Louisiana State University

LSU Digital Commons

Faculty Publications

Department of Physics \& Astronomy

$1-1-1997$

\title{
Phase-locked high-order harmonic sources
}

\author{
Raoul Zerne \\ Lunds Tekniska Högskola \\ Carlo Altucci \\ Lunds Tekniska Högskola \\ Marco Bellini \\ LENS - European Laboratory for Non-Linear Spectroscopy \\ Mette B. Gaarde \\ Lunds Tekniska Högskola \\ T. W. Hänsch \\ LENS - European Laboratory for Non-Linear Spectroscopy
}

See next page for additional authors

Follow this and additional works at: https://digitalcommons.Isu.edu/physics_astronomy_pubs

\section{Recommended Citation}

Zerne, R., Altucci, C., Bellini, M., Gaarde, M., Hänsch, T., L'huillier, A., Lyngå, C., \& Wahlström, C. (1997). Phase-locked high-order harmonic sources. Physical Review Letters, 79 (6), 1006-1009. https://doi.org/ 10.1103/PhysRevLett.79.1006

This Article is brought to you for free and open access by the Department of Physics \& Astronomy at LSU Digital Commons. It has been accepted for inclusion in Faculty Publications by an authorized administrator of LSU Digital Commons. For more information, please contact ir@lsu.edu. 


\section{Authors}

Raoul Zerne, Carlo Altucci, Marco Bellini, Mette B. Gaarde, T. W. Hänsch, Anne L'huillier, Claire Lyngå, and C. G. Wahlström 


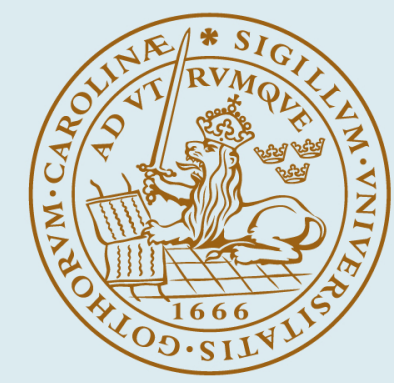

\section{LUND UNIVERSITY}

\section{Phase-locked high-order harmonic sources}

Zerne, R; Altucci, C; Bellini, M; Gaarde, M. B; Hansch, T. W; L'Huillier, Anne; Lynga, C; Wahlström, Claes-Göran

Published in:

Physical Review Letters

DOI:

10.1103/PhysRevLett.79.1006

1997

Link to publication

Citation for published version (APA):

Zerne, R., Altucci, C., Bellini, M., Gaarde, M. B., Hansch, T. W., L'Huillier, A., Lynga, C., \& Wahlström, C-G. (1997). Phase-locked high-order harmonic sources. Physical Review Letters, 79(6), 1006-1009. https://doi.org/10.1103/PhysRevLett.79.1006

\section{Total number of authors:}

8

\footnotetext{
General rights

Unless other specific re-use rights are stated the following general rights apply:

Copyright and moral rights for the publications made accessible in the public portal are retained by the authors and/or other copyright owners and it is a condition of accessing publications that users recognise and abide by the legal requirements associated with these rights.

- Users may download and print one copy of any publication from the public portal for the purpose of private study or research.

- You may not further distribute the material or use it for any profit-making activity or commercial gain

- You may freely distribute the URL identifying the publication in the public portal

Read more about Creative commons licenses: https://creativecommons.org/licenses/

Take down policy

If you believe that this document breaches copyright please contact us providing details, and we will remove

access to the work immediately and investigate your claim.
} 


\title{
Phase-Locked High-Order Harmonic Sources
}

\author{
Raoul Zerne, ${ }^{1}$ Carlo Altucci, ${ }^{1}$ Marco Bellini, ${ }^{2}$ Mette B. Gaarde, ${ }^{1,3}$ T. W. Hänsch, ${ }^{2,4}$ Anne L'Huillier, ${ }^{1}$ \\ Claire Lyngå, ${ }^{1}$ and C.-G. Wahlström ${ }^{1}$ \\ ${ }^{1}$ Department of Physics, Lund Institute of Technology, P.O. Box 118, S-221 00 Lund, Sweden \\ ${ }^{2}$ L.E.N.S, Largo E. Fermi, 2, I-50125 Florence, Italy \\ ${ }^{3}$ Niels Bohr Institute, Ørsted Laboratory, 2100 Copenhagen, Denmark \\ ${ }^{4}$ Max-Planck-Institut für Quantenoptik, P.O. Box 1513, D-85740 Garching, Germany
}

(Received 12 February 1997)

\begin{abstract}
We demonstrate that two harmonic sources generated independently in a xenon gas jet using the same picosecond Nd:YAG laser are locked in phase. The experiment is performed by separating a laser beam into two parallel beams focused at different locations under the nozzle of a gas jet, and therefore producing two independent sources of harmonic radiation, and studying the pattern obtained in the far field when the radiations from these sources interfere. A high and robust fringe visibility is obtained. [S0031-9007(97)03735-6]
\end{abstract}

PACS numbers: $32.80 . \mathrm{Rm}, 42.65 . \mathrm{Ky}$

High-order harmonic generation $(\mathrm{HG})$ in gases has attracted considerable attention in the last few years, motivated by the potential of the harmonic radiation to become a useful coherent source in the extreme ultraviolet (XUV) range. Although fundamental aspects of HG have been extensively studied [1], many issues regarding the use of harmonics as a practical source of high-frequency radiation remain to be explored. Efforts are being made in improving and measuring the conversion efficiency [2], in extending the available energy range $[3,4]$, and in characterizing the properties of the harmonic radiation, spatial coherence [5], angular emission [6-8] and pulse duration $[9,10]$. In the present work, we investigate aspects related to the temporal coherence of the harmonic radiation. More precisely, we study whether we can create two phase-locked sources of harmonic radiation, with enough coherence so that when the two beams overlap in the far field, an interference pattern is created.

To create "phase-locked" harmonic sources might seem trivial at first glance: Harmonic generation is a coherent process and there should be a well-defined phase relation between the generated and the driving fields. This implies that two sources of harmonics, created by two pulses originating from the same laser, should be locked in phase. However, theoretical studies of the single-atom response of an atom exposed to a strong laser field show that the phase of the high-order harmonics varies rapidly with the laser intensity [11]. Since the atom experiences different intensities as a function of time during the laser pulse, the intensity-dependent phase is expected to lead to a frequency chirp of the generated field [11,12], which might obscure this phase locking. Only if the intensities in the two beams are exactly matched at every instant during the laser pulse are the two harmonic sources expected to have a constant phase relationship during the pulse, and hence be mutually coherent. The motivation for the present Letter is to demonstrate the phase locking between two harmonic sources created by the same laser and to study its sensitivity with respect to differences in the intensities of the driving fields for the two sources.

Our experimental setup presents some analogies with Young's double slit experiment. We create harmonics in two different foci separated by a small distance and observe the interference pattern in the far field. Note the fundamental difference with Young's double slit experiment: The radiations from the two sources are not directly emerging from the same plane wave (and therefore locked in phase), but are generated through the process of high-order harmonic generation. The experiments are performed using a picosecond laser system focused in a jet of xenon atoms. We find that the generated harmonics, from the 7 th to the 17 th, lead to a good and robust fringe visibility. We have performed detailed calculations simulating the experimental conditions and find that intensitydependent phase shifts can indeed remain negligible, well into the plateau region of high-order harmonic generation, i.e., into a very interesting wavelength range in the extreme ultraviolet.

We begin by introducing some notations, which are similar to those used to describe Young's double slit experiment. We consider two harmonic sources of radiation separated by a distance $\delta$ and measure the interference pattern in an observation plane placed at a distance $L$ from the sources. In the ideal case, where the sources are perfectly phase locked, quasimonochromatic, with no intensity-dependent phase and no chirp, the intensity of the total field at a point with ordinate $Y$ in the observation plane reads as

$$
I=I_{1}+I_{2}+2 \sqrt{I_{1} I_{2}} \cos \left(2 \pi \frac{\delta Y}{\lambda L}\right)
$$

where $I_{1}, I_{2}$ are the intensities of the two harmonic beams at the same point and $\lambda$ the harmonic wavelength. The visibility, or contrast of the fringes, defined by $V=$ $\left(I_{\max }-I_{\min }\right) /\left(I_{\max }+I_{\min }\right)$ is, in this simple case, equal to $2 \sqrt{I_{1} / I_{2}} /\left(1+I_{1} / I_{2}\right)$. $V$ is equal to 1 when the 
harmonic pulses have the same intensity and decreases with the harmonic intensity ratio. How rapidly $V$ decreases with the intensity ratio of the two fundamental pulses reflects, in this ideal case, how rapidly the harmonic strength varies with the laser intensity.

For a generation process inducing a frequency chirp, i.e., a temporal variation of the instantaneous frequency during the laser pulse, however, the decrease of the visibility with the intensity ratio depends also on how rapidly the harmonic phase varies with the laser intensity. The instantaneous fringe pattern, at any given time $(t)$ during the laser pulse, reads as

$$
\begin{aligned}
I(t)= & I_{1}(t)+I_{2}(t)+2 \sqrt{I_{1}(t) I_{2}(t)} \\
& \times \cos \left[2 \pi \frac{\delta Y}{\lambda L}+\phi_{1}(t)-\phi_{2}(t)\right],
\end{aligned}
$$

where $\phi_{1}(t), \phi_{2}(t)$ are the phases of the generated fields at the observation point at time $t$. The observed interference pattern is obtained by integrating $I(t)$ over the pulse duration. If $\phi_{1}(t)-\phi_{2}(t)$ varies significantly during the laser pulse (i.e., by more than $\approx \pi$ over the time during which harmonics are generated), the fringe pattern will be washed out upon time integration.

The experimental setup is shown in Fig. 1. The output from a mode-locked Nd:YAG laser (1064 nm, $35 \mathrm{ps)} \mathrm{[13]}$ is focused by a $255 \mathrm{~mm}$ focal length lens $(f \#=30)$ and then split into two spatially displaced parallel beams of equal intensities but orthogonal polarizations in a beta barium borate (BBO) crystal. The axes of the crystal are oriented at $45^{\circ}$ with respect to the laser polarization. A polarizer placed after the crystal transmits a common polarization component in the two beams. By rotating the polarizer, the intensity ratio between the two beams can be continuously varied. The distance between the two beams, and consequently between the two foci, is $\delta \approx$ $150 \mu \mathrm{m}$, while the beam waist diameter in each focus is less than $50 \mu \mathrm{m}$. The two sources of harmonic radiation are hence well separated in space and independent of each other. The maximum intensity in each of the two foci is estimated to be slightly above $10^{13} \mathrm{~W} / \mathrm{cm}^{2}$. Harmonic radiation of a particular order (ranging between the 7 th and the 17th) is selected by a normal-incidence spherical

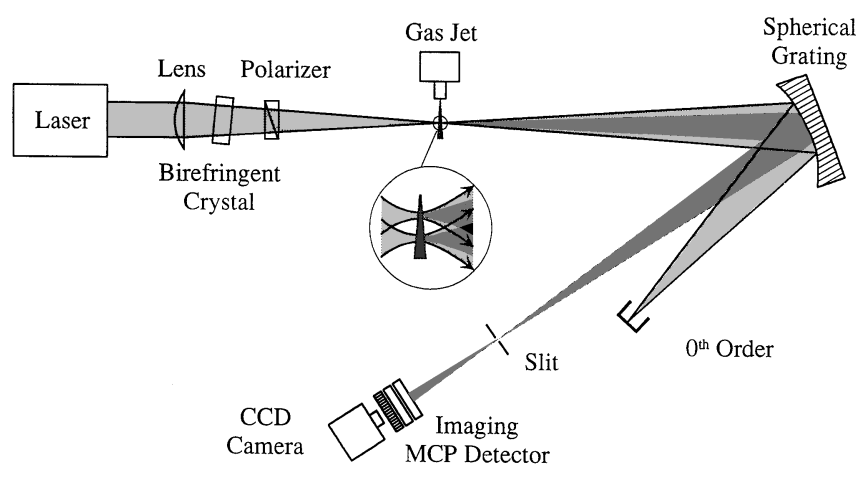

FIG. 1. Experimental setup. grating and imaged onto a micro-channel-plate detector (MCP), coupled to a charged-coupled device camera. The distance $(L)$ from the focal plane of the spectrometer and the observation plane is about $75 \mathrm{~cm}$.

An example of a recorded far-field pattern for the 13th harmonic is shown in Fig. 2(a). The intensities in the two beams are approximately equal. Interference fringes are clearly visible. Their position is very stable on a
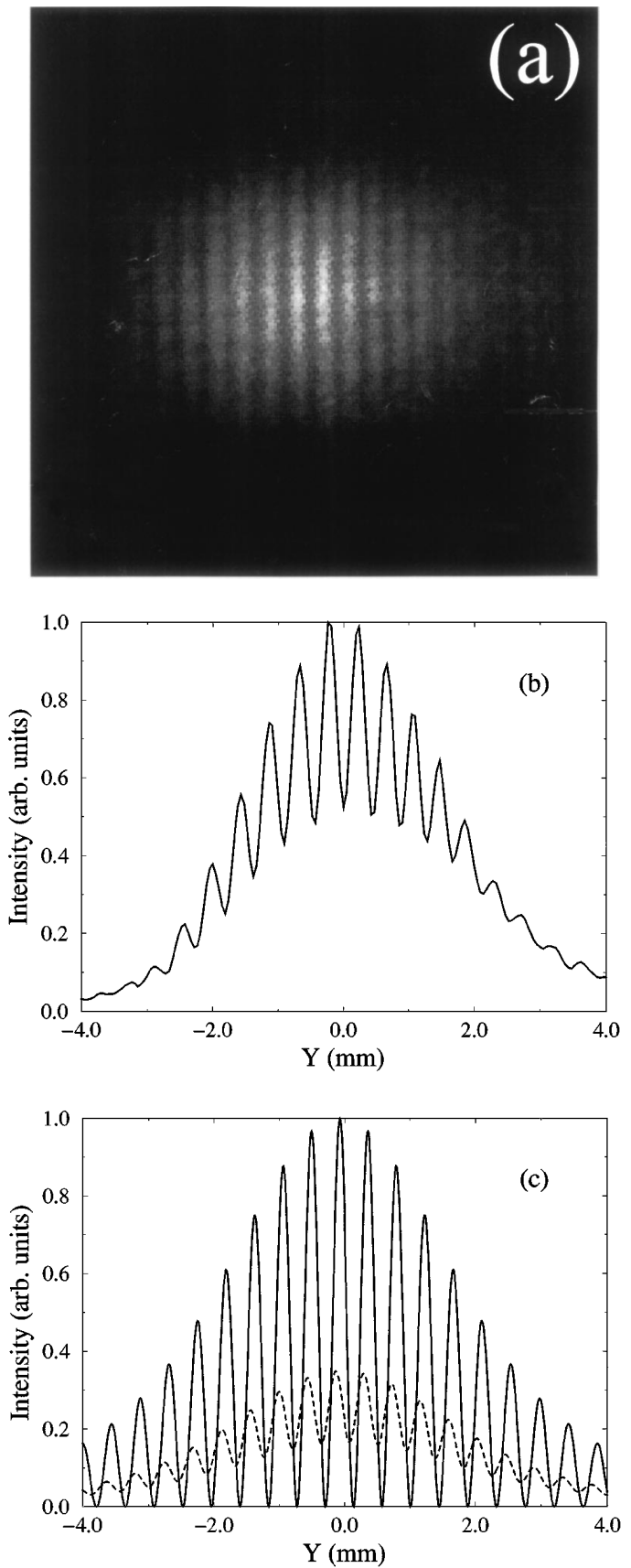

FIG. 2. (a) Interference pattern for the 13th harmonic for equal intensities of the two laser pulses, (b) integration of the fringe pattern shown in (a), and (c) theoretical simulation in the same conditions. The solid line has been obtained for equal intensities, the dashed line for an intensity ratio of 0.75 . 
shot-to-shot basis. The recording has been obtained by integrating over 30 laser shots. However, similar results are obtained in single-shot recordings as well. Figure 2(b) shows the integration of the fringe pattern in the vertical direction. The contrast of the fringes is $30 \%$. The fringe interval is measured to be $0.44 \mathrm{~mm}$, which agrees with the expected interval $i=\lambda L / \delta=0.41 \mathrm{~mm}$. We find that the interference pattern is rather robust, and that it can be observed, with varying degrees of contrast, even when the peak intensities in the two foci differ by almost a factor of 2. It is also observed to be independent of the position of the gas jet relative to the laser focus, over the length over which harmonics can be observed (a few $\mathrm{mm}$ ). In Fig. 3, we show two fringe patterns obtained with the 9th harmonic. The fringe interval is larger than for the 13th harmonic, $0.6 \mathrm{~mm}$, directly reflecting the longer wavelength. Figure 3(a) is obtained with approximately equal intensities for the two laser pulses, while in Fig. 3(b) the laser intensity ratio is about 0.6 . The contrast decreases in this case from $30 \%$ to $6 \%$. The contrast is never observed to be better than $\sim 30 \%$ for single-shot recordings
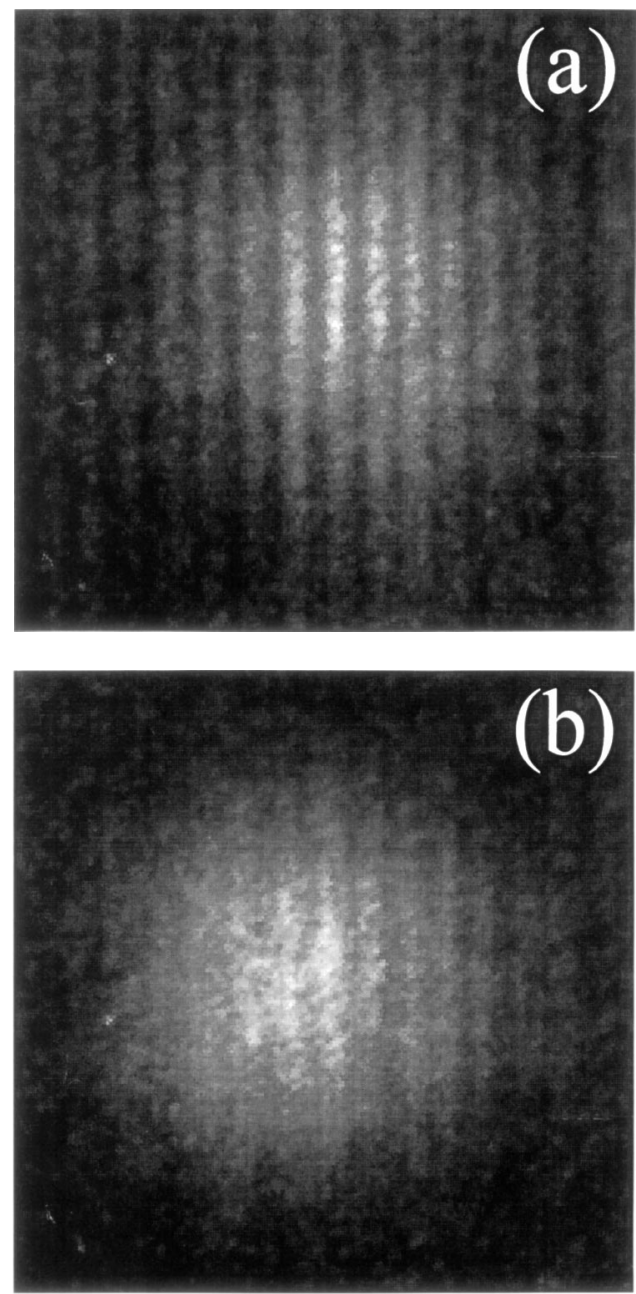

FIG. 3. Interference patterns for the 9th harmonic for (a) equal laser intensities and (b) an intensity ratio equal to 0.6. and does not improve significantly with averaging. Some of the laser shots, apparently in a random fashion, do not lead to any fringe pattern: we believe this might reveal some instability of the driving laser itself. Unfortunately, this prevents us from performing systematic quantitative measurements, e.g., of the contrast as a function of the laser intensity ratio.

To interpret the experimental results, we have performed numerical simulations for conditions close to the experimental ones. The method is described in a previous Letter [11]. It uses the strong field approximation for the single-atom response and the slowly varying envelope approximation for the propagation of the generated field. The fringe pattern is obtained by numerically superposing, in the far field, the two harmonic fields generated in each focus. The result obtained for the 13th harmonic is shown in Fig. 2(c). The solid line shows the result for equal intensities of the two laser pulses $\left(1.5 \times 10^{13} \mathrm{~W} / \mathrm{cm}^{2}\right)$; the curve in the dashed line is obtained for an intensity ratio of 0.75 . The theoretical results compare well with the experimental one. The fringe interval and the total number of fringes observed (which depends on the harmonic angular distribution) are very similar. The contrast is equal to 1 for equal intensities, as expected, and decreases with the intensity ratio (it is $30 \%$ for an intensity ratio of 0.75 ). In Fig. 4, we examine the variation of the total phase for the 13th harmonic field in xenon as a function of time for two different peak intensities: $0.75 \times 10^{13}$ (dotdashed line) and $1.5 \times 10^{13} \mathrm{~W} / \mathrm{cm}^{2}$ (solid line). We consider a point in the observation plane on axis. The phase variation of the propagated field is found to be approximately quadratic. The total phase variation $\phi_{1}(t)-\phi_{2}(t)$ over $30 \mathrm{ps}$ is about $0.8 \mathrm{rad}$. This does not induce a significant displacement of the fringe pattern. In Fig. 5, we plot the contrast as a function of the laser intensity ratio, using a maximum intensity of $1.5 \times 10^{13} \mathrm{~W} / \mathrm{cm}^{2}$ (full squares). As in the experiment, the contrast decreases rather slowly with the intensity ratio. The influence of the

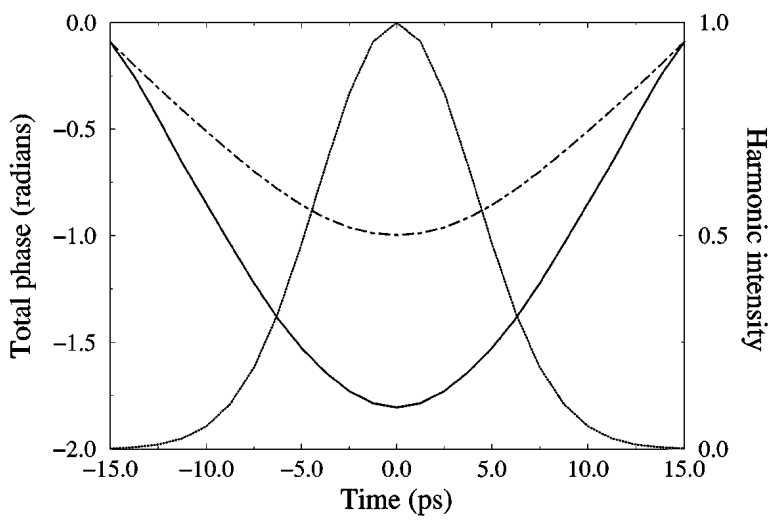

FIG. 4. Calculated phase variation for the 13th harmonic in xenon at peak intensities of 1.5 (solid line) and $0.75 \times$ $10^{13} \mathrm{~W} / \mathrm{cm}^{2}$ (dot-dashed line). The harmonic intensity is indicated by the dashed line. 


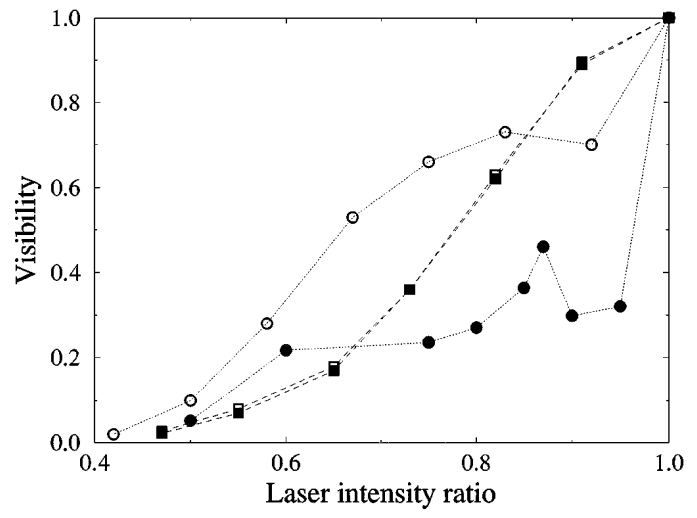

FIG. 5. Fringe contrast as a function of laser intensity ratio for the 13th harmonic in xenon (solid squares) and for the 43rd harmonic in neon (solid circles). The curves with the empty symbols (squares for the 13th harmonic and circles for the 43rd harmonic) are obtained without accounting for the intensitydependent phase variation.

intensity-dependent phase variation is shown by comparing the full squares to the empty ones, obtained by setting artificially $\phi_{1}(t)=\phi_{2}(t)$ in Eq. (2). As expected from Fig. 4, the effect of the phase variation is practically negligible. These numerical data are in very good agreement with the experimental results and give a rather simple explanation to the phase locking: the intensity-dependent phase variation is not important enough, in the cases investigated, to induce a significant chirp of the harmonic fields.

In order to complement these results, we have also performed numerical simulations in conditions where a significant frequency chirp of the generated radiation has been predicted [11,12]. Such conditions are realized in the case of the 43rd harmonic, of $150 \mathrm{fs}, 790 \mathrm{~nm}$ radiation, generated in neon at a maximum intensity of $5 \times 10^{14} \mathrm{~W} / \mathrm{cm}^{2}$. The results are shown by the circles in Fig. 5. As expected, in contrast to the 13th harmonic in $\mathrm{Xe}$, there is a significant effect of the phase variation. Consequently, the contrast decreases rapidly with the intensity ratio. This is due to the displacement of the fringe pattern during the pulse, induced by the intensity-dependent frequency chirp. It might therefore be difficult to obtain, experimentally, robust fringe patterns by superposing two high-order harmonic fields. However, by reducing as much as possible the linear chirp [14], which can be done by optical techniques, or, possibly, by imparting to the fundamental pulse a chirp with opposite sign, most of the phase variation should be eliminated, and a more robust fringe pattern could be obtained.

A direct application of the phase locking is the generation of coherent XUV radiation whose degree of polariza- tion can be continuously controlled. This can simply be achieved in the present setup by removing the polarizer after the BBO crystal (see Fig. 1), so that the two generated harmonic beams are cross polarized and the total field has a degree of ellipticity varying from circular to linear in space. To check this idea, in addition to removing the polarizer, we place an XUV polarizer (a gold-coated mirror at a $45^{\circ}$ angle of incidence) before the MCP detector. We indeed obtain interference patterns similar to the ones shown in Figs. 2 and 3. By placing a slit perpendicular to the direction defined by the two foci, it is possible to obtain XUV radiation with a given degree of polarization.

In conclusion, we have demonstrated that harmonics of "moderately" high orders, such as those produced by picosecond laser systems at relatively modest intensities, are coherent, in the sense that they lead to stable interference patterns. This makes it possible to split the laser beam into two and to generate two independent but phase-locked beams at the new frequency, which can be directly used, for example, in an interferometry experiment. This result opens perspectives for applications such as polarimetry, interferometry, holography, or even high resolution Ramsey spectroscopy in this short wavelength region.

We acknowledge the support of the Swedish Natural Science Research Council and the EC "Access to Large Scale Facilities" Programme (Contract No. ERBFMGECT950020). Fruitful discussions with Demetris Lappas and Misha Ivanov are gratefully acknowledged.

[1] See contributions in Proceedings of the 7th International Conference on Multiphoton Processes, Garmisch-Partenkirchen, Germany, 1996, edited by P. Lambropoulos and H. Walther, Institute of Physics Conference Series Vol. 154 (Institute of Physics Publishing, Philadelphia, 1996).

[2] T. Ditmire et al., Phys. Rev. A 51, R902 (1995).

[3] J. Zhou et al., Phys. Rev. Lett. 76, 752 (1996).

[4] S. G. Preston et al., Phys. Rev. A 53, R31 (1996).

[5] T. Ditmire et al., Phys. Rev. Lett. 77, 4756 (1996).

[6] P. Salières et al., J. Phys. B 29, 4771 (1996).

[7] J. Peatross and D. D. Meyerhofer, Phys. Rev. A 51, R906 (1995).

[8] J. W. G. Tisch et al., Phys. Rev. A 49, R28 (1994).

[9] T. E. Glover et al., Phys. Rev. Lett. 76, 2468 (1996).

[10] J. M. Schins et al., J. Opt. Soc. Am. B 13, 197 (1996).

[11] P. Salières et al., Phys. Rev. Lett. 74, 3776 (1995).

[12] C. Kan et al., Phys. Rev. A 52, R4336 (1995).

[13] S. Svanberg et al., Phys. Scr. 49, 187 (1994).

[14] K. J. Schafer and K. C. Kulander, Phys. Rev. Lett. 78, 638 (1997). 